\title{
Incorporación de harina de polen en panificación
}

\section{Incorporation of pollen flour in baking's}

\author{
Martin Lazcano-Hernández*1; Addi Rhode Navarro-Cruz ${ }^{2}$; Raúl Ávila Sosa Sanchez ; José Alejandro \\ Hernandez-Abundez ${ }^{4}$; Cintia Abigail Zerón-Alvarado ${ }^{5}$ : Daniel Santiago Pereira ${ }^{6}$
}

${ }^{1}$ Maestro en Ciencias, Catedrático Facultad Ciencias Químicas, Benemérita Universidad Autónoma de Puebla, Puebla, México, lazmar@ gmail.com; ²Doctor en Ciencias, Universidad Complutense, Catedrático en Facultad Ciencias Químicas, Benemérita Universidad Autónoma de Puebla, México, addi.navarro@correo.buap.mx; ${ }^{3}$ Doctor en Ciencias, Catedrático en Facultad Ciencias Químicas, Universidad de las Américas Puebla, México, raul.avila@correo.buap.mx; ${ }^{4}$ Colaborador en Laboratório de Alimentos en Facultad de Ciencias Químicas, Puebla, México, alexma936@gmail.com; ${ }^{5}$ Colaborador en Laboratório de Alimentos en Facultad de Ciencias Químicas, Benemérita Universidad Autónoma de Puebla, Puebla, México, alixceron@gmail.com; ${ }^{6}$ Doctor en Ciencia Animal, Investigador en Laboratório de Agroindústria da Embrapa Amazônia Oriental, Belém, Pará, Brasil, daniel.pereira@embrapa.br.

\section{A R T I G O}

Recebido: 06/05/2018

Aprovado: 07/12/2018

Palabras Clave:
Harina de polen
Pan blanco
Panificación

Key words:

Pollen flour

Bread

Banking.

\section{R E S U M E N}

Todos los días invariablemente un alimento de consumo popular en la mesa semanal y a cualquier hora del día es el pan, actualmente para sectores de la población la modificación en sabores, texturas y composición poseen auge en el comercio. La sustitución de la harina de trigo por diferentes cereales, leguminosas o ingredientes funcionales abarcan espacio en panadería tradicional como en las panaderías del centro comercial. El polen de abeja es un alimento saludable ya que posee 30-35 \% de proteína en forma de aminoácidos esenciales por arriba del patrón. Sus capas de ectina y exina permiten al polen no ser tan digerible al ser humano, por lo que un producto horneado favorecería su aprovechamiento. En esta investigación se va a establecer la formulación adecuada para integrar la harina de polen a un pan blanco de masa salada hasta donde sensorialmente no se detecte la adición, así como estandarizar la metodología de panificación. Tomando en cuenta parámetros como: Cantidad de harina de trigo-polen y azúcar, tiempo de fermentación, tiempo de horneo y temperatura de horneo. El producto obtenido es valorado sensorialmente para determinar la concentración de harina y cuál es el porcentaje de adición mejor evaluado sensorialmente, el rango de adición aceptado es de 5 a $10 \%$ de sustitución de harina de trigo. Sensorialmente no existe diferencia en atributos como color, olor, sabor, consistencia y textura en este rango, siendo ligeramente mejor el sabor a $10 \%$ de Harina de polen. No obstante el pan blanco obtenido se puede comercializar hasta $10 \%$ de adición, siendo alternativa de los apicultores para obtener ganancias extras no solo de la venta de miel.

\section{A B S T R A C T}

Every day invariably a popular consumption food in the weekly table and at any time of the day is bread, currently for sectors of the population the change in flavors, textures and composition have boom in the trade. The substitution of wheat flour with different cereals, legumes or functional ingredients encompasses space in traditional bakeries as well as bakeries in the commercial center. Bee pollen is a healthy food because it has $30-35 \%$ protein, is found in the form of essential amino acids above the standard. Its layers of ectin and exine allow the pollen not to be so digestible to the human being, so a baked product would favor its use. This research will establish the appropriate formulation to integrate the flour of pollen to a bread dough until where sensorially the addition is not detected, as well as to standardize the methodology of bakery. Taking into account parameters such as: quantity of flour wheat-pollen and sugar y/o honey, fermentation time, baking time and baking temperature. The product obtained is sensorially valued to determine the concentration of flour and which is the percentage of addition best evaluated sensory, the accepted range of addition is 5 to $10 \%$ substitution of wheat flour. Sensorially there is no difference in attributes such as color, odor, flavor, consistency and texture in this range, being slightly better the flavor of $10 \%$ of pollen flour. However the white bread obtained can be marketed up to $10 \%$ of addition, being alternative of the beekeepers to obtain extra profits not only from the sale of honey.

\section{Revista Verde}

ISSN 1981-8203

Pombal, Paraíba, Brasil v. 14, n.1, jan.-mar, p.48-54, 2019

doi: $10.18378 /$ rvads.v14i1.5920 


\section{INTRODUCIÓN}

De acuerdo a las proyecciones de la Organización 'para la Cooperación y el Desarrollo Económico (OCDE) y la Organización de las Naciones Unidas para la Agricultura y la Alimentación (FAO) para el año 2024/2025 se producirán al menos 2,800 millones de toneladas de cereales en el mundo, es decir, 294 millones de toneladas más que en el año 2015, un crecimiento acumulado del $12 \%$ en el periodo. En cuanto al trigo, se prevé que su producción supere los 787 millones de toneladas, 63 millones más que el año 2015, destacando los incrementos en millones de toneladas por parte de la India (13), la Federación de Rusia (10), la Unión Europea (8), China (5), Argentina (4) y Paquistán (4) (ESPIGUERO, 2016; SAGARPA, 2016).

El trigo es el cereal más utilizado en la alimentación humana, debido al alto valor energético y a un mayor contenido de proteínas en comparación con el maíz y el arroz. A medida que la demanda de carne y productos lácteos aumente, también lo hará en proporción la producción de cereales secundarios y pasta de soya por su vínculo en la alimentación animal. En el caso del trigo se estima un crecimiento en su consumo para la formulación de alimentos de uso forrajero en China, la Federación Rusa y la Unión Europea. El Trigo como cereal sigue avanzando en el camino que lo consolida como un alimento esencial para el mundo, cada vez con más impacto en los humanos en su consumo, a través de productos especializados y en la formulación de alimentos de consumo animal. Debido a que la producción de trigos panificables continua siendo insuficiente para abastecer la demanda de la industria molinera nacional, para este mismo periodo se importaron 4 millones 356 mil toneladas, 3\% menos que el año agrícola previo (CANIMOLT, 2015).

Durante el ciclo 2015/2016 la importación aumentó 4 millones 461 mil toneladas, equivalente al $2.4 \%$. En cuanto al trigo cristalino o durum, el mercado nacional requiere alrededor de 720 mil toneladas para consumo humano, por lo que el excedente de producción se destina, a la exportación como un mercado de oportunidad y al sector pecuario como un mercado consumidor de excedentes. El consumo nacional aparente de granos y oleaginosas en México para 2015 ascendió a 39.9 millones de toneladas de las cuales 22.7 Millones de toneladas, fueron importaciones por un valor superior a los 6 mil millones de dólares (SAGARPA, 2013, 2015, 2016; CANIMOLT, 2015; CANAINPA, 2016).

El pan desde la antigüedad siempre se ligó a la alimentación humana sufriendo modificaciones como toda evolución, la fabricación ha sido preferentemente artesanal o como empresa familiar. El consumo per cápita es de $34 \mathrm{~kg}$ en México siendo el consumo de pan blanco en $70-75 \%$ con ventas anuales de 64 mil 320 millones de pesos el resto corresponde a pan dulce, galletas, pasteles, etc., Se estima su valor como industria panificadora en 6, 500 millones de Dólares repartidos en 58 mil 622 establecimientos que incurren en ventas o en la elaboración del ramo el cual se distribuye principalmente en panaderías $(68 \%)$, pastelerías $(18 \%)$, tortillerías $(12 \%)$, pero como cadena industrial es la tercera empleadora de mano de obra al predominar la forma artesanal.

en producir (ANPROPAN, 2016; CANIMOLT, 2016; CANAINPA, 2015, 2016).

El país con mayor consumo por persona y año lo ostenta Turquía con 104 kilos, seguido de Serbia y Montenegro con
98 kilos, Bulgaria con 95 kilos, Ucrania con 89 kilos y Arabia Saudí con 86 kilos. Fuera de Europa, también según datos de la UIB, Chile encabeza el consumo con 96 kilos por persona y año, seguido de Argentina con 76 kilos, Estados Unidos con 43 kilos, Ecuador con 37, México con 33, Costa Rica, con 32 kilos, Brasil y Perú con 31 kilos, Colombia con 22 y Venezuela con 19 kilos. Japón llega a los 22 kilos por persona y año, China apenas alcanza los seis kilogramos per cápita. El mercado nacional e internacional en panificación, hoy demanda de harina de kamut, escanda o espelta, quínoa y una creciente petición de panes de centeno, semillas, nuevos cereales, pero todos ligados a las palabras artesanía, tradición, masa madre, lentas y largas fermentaciones, así como sabores ligados al territorio o la zona geográfica (MURCIA, 2017).

El polen de abeja es un material de consistencia parecida a la del polvo en forma de pelotitas, que producen las anteras de las plantas en florescencia y que las abejas recogen como su alimento en tiempo de escases. Los entusiastas del polen de abeja lo solicitan como un-alimento perfecto-posee 30-35 $\%$ de proteína, vitaminas del complejo $\mathrm{B}$, vitamina $\mathrm{C}$, aminoácidos, ácidos grasos esenciales, enzimas, caroteno, calcio, cobre, hierro, magnesio, potasio, manganeso, sodio, esteroles vegetales y azúcares simples. Casi la mitad de su proteína se encuentra en forma de aminoácidos disponibles, listos para usarse directamente por el cuerpo y como tal su proteína es asimilable para en excelente crecimiento. El polen es considerado un tónico vigorizante y nutritivo en la medicina china, mejora la resistencia y la vitalidad, ayuda a la recuperación de enfermedades crónicas, añade más peso a la persona convaleciente, reduce los antojos y adicciones, normaliza los intestinos, previene las enfermedades transferibles como el resfrío común y la gripe, al igual que otros productos de las abejas, el polen tiene un efecto antibacteriano. También es útil para las personas que tienen alergias, pues fortalece el sistema inmunológico (OPENSHAW, 2015).

El polen de abeja fresco no debe ser pegajoso ni debe formar grumos y tiene que envasarse en recipientes muy bien sellados. Algunas personas (se calcula que el 0.05 por ciento de la población) son alérgicas al polen de abeja. Se recomienda empezar con una cantidad pequeña observando si se presenta sarpullido, dificultad respiratoria, molestia o algún tipo de reacción. Si se presenta algún síntoma, se debe suspender el consumo. La dosis óptima de polen varía con las necesidades personales. Para la prevención de alergias, por ejemplo, 6 gramos diarios en forma de tabletas, cápsulas, o gránulos sueltos es con frecuencia suficiente. Los atletas que deseen fuerza y resistencia pueden ingerir de 10-15 gramos o más al día (KRYSTIJHAN et al., 2018; CONTE et al., 2018; KIELISZEK et al., 2018).

En los hogares mexicanos por estrato socioeconómico en cuanto a gasto en pan blanco se distribuyen en nivel bajo $(10 \%)$, nivel medio bajo (55\%) y medio alto (28\%) además del alto $(7 \%)$ dada su versatilidad como alimento se puede utilizar para ser acompañado de ingredientes dulces así como también un alimento principal en las conocidas tortas o molletes, alimento popular a cualquier hora del día en toda la república mexicana, cuyo precio al aumentar no reduce su volumen de venta ya que la población tiene por costumbre ser utilizados en reuniones y celebraciones, del sector alimentos se mantiene en operación un $50 \%$ de su capacidad instalada superando la media general de la industria con un $63 \%$. El crecimiento de panaderías dentro de las tiendas de 
autoservicio se establece nuevos modelos de negocio con mejores servicios del pan, incorporando ingredientes funcionales, harinas de diversas semillas o leguminosas, sabores, olores y condimentos (MURCIA, 2017; CANIMOLT, 2016; CANAINPA, 2016; ESPIGUERO, 2016; MURCIA, 2017; INDUSTRIA ALIMENTARIA, 2015).

Debido que el polen presenta propiedades nutricionales de beneficio a la salud para el ser humano, se propone integrar el polen en forma de harina a un producto de panificación como el pan blanco en forma de torta estableciendo la formulación mediante la aceptación sensorial de un producto que sea similar con el comercial.

\section{MATERIAL Y METODOS}

\section{Materias Primas: Polen y miel}

El ingrediente adicionado al producto a obtener es harina de polen procedente de la cosecha primavera-verano, multiflora del Estado de Aguascalientes, México. El cual en forma de grano es molido con malla 60 para homogeneizar el polvo obtenido y de esta forma es incorporado como ingrediente. La miel se obtuvo del mismo estado, con cosecha otoño-invierno, multiflora, semisólida, esta fue diluida en agua 1:3 y de esta forma se hidrata la mezcla para elaborar el pan.

\section{Formulación del pan blanco}

Metodológicamente se parte de una formulación base para masa salada, un pan blanco como es la torta; como ingredientes lleva Harina de trigo, levadura en polvo, azúcar, sal, margarina, agua la suficiente para obtener una masa elástica y uniforme. El procedimiento seguido es cernir la harina, se forma un volcán en el centro de la harina se agrega la levadura después la sal y a continuación, se le agrega 8$10 \mathrm{ml}$ de agua tibia hasta obtener una mezcla homogénea. Y se comienza a amasar hasta eliminar los grumos de la harina, agregando azúcar por último la mantequilla no dejar de amasar hasta obtener una masa plástica y uniforme. Se deja reposar 30 minutos a $40^{\circ} \mathrm{C}$ en una fermentadora. Se le da forma al pan como torta, aunque puede ser bolillo o telera. Una vez fermentada la masa se moldea por pieza, se barniza con la yema de huevo dejándose en reposo por 10 minutos a $40^{\circ} \mathrm{C}$. El horno debe precalentarse hasta alcanzar una temperatura aproximada de $200^{\circ} \mathrm{C}$ en su interior, posteriormente se mete en charolas al horno hasta que tome un color café en la superficie del mismo.

Verificar al incrusta el cuchillo el cual deberá salir sin masa cruda en el centro, sacar del horno y dejar enfriar el pan. Existe una modificación a la técnica se adiciona la miel con agua en forma de jarabe, junto con la levadura, azúcar, sal en la harina para formar la masa más una cantidad suficiente de agua para formar una masa plástica, uniforme y húmeda pero moldeable, se hornea a $190^{\circ} \mathrm{C}$ por 30 minutos. Al principio solo se agregó un $50 \%$ de harina de polen, pero se observó que el pan tenía un sabor amargo, una mala consistencia de la masa, así como una textura dura después de hornear por lo que se fueron realizando modificaciones para mejorar el sabor, aspecto y textura. Las variables con la que se experimentó son; Harina de trigo (93-95g), Harina de polen (5-10g), jarabe de miel $(10-14 \mathrm{ml})$ con agua $(30-37 \mathrm{ml})$, así como tiempo de horneo, tiempo de fermentación en la primera y segunda acción, hasta ajustar la formulación adecuada sensorialmente para la torta estableciendo dichos parámetros para la formulación de mezclas por el método de multivariables de Zhu (AVILÉS-SACOTO et al., 2015;
IMANIRAD et al., 2015; FLECHA, 2015; MARTINEZ et al., 2017; SALA; HAROS, 2016).

\section{Determinación sensorial para formular y conocer la mayor adición aceptada de harina de polen.}

La forma del pan blanco utilizada es la torta y el bolillo por ser las más comunes en el consumo de la población. Sensorialmente se valora al producto llevándose a cabo con panelistas no entrenados en la Facultad de Ciencias Químicas a mediodía, sin distinción de género, raza o religión. Criterios de inclusión: Edad entre 19-23 años, hombre o mujer, curse la carrera de QFB. Criterios de exclusión; fuera del rango de edad, fumar o tomar antes de la prueba, haber probado alimento como mínimo 4 horas antes de la prueba. La prueba de preferencia bajo los anteriores criterios se llevó a cabo con personas $(n=30)$ para conocer si gusta o no sensorialmente de manera general el pan elaborado en una escala estructurada de 5 puntos, numérica, unimodal del 1 a 5 determinando que tanto gusta de aceptación general. En una primera instancia se formula en base a un pan normal con la sustitución de un porcentaje de harina de polen 10 hasta $50 \%$ para evaluar el pan horneado. A mayor cantidad de polen se observa menor calidad de pan sensorialmente, en prueba de preferencia para el producto formulado se le realiza un ajuste al adicionar un jarabe miel-agua para aumentar la aceptación y modificar el sabor amargo de polen en el pan. La prueba estableció un rango de 5 a $10 \%$ de adición de la harina de polen con características sensoriales aceptables.

Para la evaluación sensorial de aceptación por atributos del producto elaborado tomando en cuenta los criterios arriba expuestos se realiza a Estudiantes de Ciencias Químicas del 1 al 10 cuatrimestre de las carreras de QFB, Químicos y Farmacia, muestras de 3 a 4 g. con porcentaje de harina de polen 5 a $7 \%$ fijando la fermentación primera en 30 minutos, 6 minutos de reposo ambiente y 25 minutos de horneo a $190^{\circ} \mathrm{C}$.

Posteriormente se procede a una evaluación sensorial de aceptación realizada con tres formulaciones las más agradables (5, 7 y $10 \%$ harina de polen) se realiza con panelistas no entrenados $(n=100)$ sin distinción de género, utilizando una boleta estructurada, numérica, bimodal de 5 puntos donde $1=$ me disgusta mucho, $3=$ no gusta ni disgusta $y$ $5=$ me gusta mucho. Se presenta la muestra al panelista no entrenado en un plato de plástico con 3 separaciones y numerados con 3 dígitos señalizándolo como sal 1, sal 2 y sal 3 con $5 \mathrm{~g}$. de muestra, además de un vaso de $10 \mathrm{ml}$ de agua simple para poder enjuagarse la boca entre muestras (ESPINOZA, 2007).

\section{RESULTADOS Y DISCUSIÓN}

El polen comercial, utilizado al molerlo presentaba dificultades al obtenerse en forma de lodo o como una masa pegajosa por lo que se le determina humedad, encontrando una humedad de $15 \%$ por lo que antes de agregarlo a la masa cada vez a utilizar en la formulación del pan se tuvo que hacer un secado a $35^{\circ} \mathrm{C}$ por 5 minutos. Al corregir la humedad con molienda malla 60 se obtuvo un granulo fino el cual se conserva en frasco de vidrio con tapa a temperatura ambiente para no permitir hidratarse de nuevo.Se procede a elaborar la masa salada basándonos en el diseño mencionado en Tabla 1 para su optimización tomando en cuenta la textura y sabor. 
Tabla 1. Diseño de formulación del pan blanco.

\begin{tabular}{|c|c|c|c|c|c|c|c|c|c|c|c|c|c|c|c|c|c|}
\hline \multirow{3}{*}{\begin{tabular}{l}
\multicolumn{1}{c}{ Muestra } \\
Harina trigo \\
Harina polen
\end{tabular}} & \multirow[t]{2}{*}{$\begin{array}{c}\begin{array}{c}\text { Sustitución } \\
\text { de harina } \\
(\%)\end{array} \\
0.00\end{array}$} & \multirow[t]{2}{*}{$\begin{array}{c}\text { Jarabe } \\
\text { Agua: Miel } \\
\text { Proporción } \\
30 / 10\end{array}$} & \multicolumn{3}{|c|}{$\begin{array}{c}\text { Tiempo } \\
\text { primer } \\
\text { fermentación } \\
40^{\circ} \mathrm{C} \text { (mins.) }\end{array}$} & \multicolumn{3}{|c|}{$\begin{array}{c}\text { Tiempo } \\
\text { segunda } \\
\text { fermentación } \\
\text { ambiente } \\
\text { (mins) }\end{array}$} & \multicolumn{3}{|c|}{$\begin{array}{c}\text { Tiempo de } \\
\text { horneado } 190^{\circ} \\
\mathrm{C} \text { (mins) }\end{array}$} & \multicolumn{6}{|c|}{ Volumen pan $(\mathrm{cm})$} \\
\hline & & & 20 & 20 & 20 & 6 & 8 & 10 & 20 & 23 & 25 & 3.5 & 3.40 & 3.4 & 3.50 & 3.50 & 3.50 \\
\hline & 5.00 & $34 / 16$ & 20 & & & 6 & 8 & 10 & 20 & 23 & 25 & 3.4 & 3.50 & 3.50 & 3.50 & 3.40 & 3.40 \\
\hline & & & & 25 & & 6 & 8 & 10 & 20 & 23 & 25 & 3.4 & 3.41 & 3.42 & 3.46 & 3.41 & 3.42 \\
\hline & & & & & 30 & 6 & 8 & 10 & 20 & 23 & 25 & 3.4 & 3.41 & 3.41 & 3.44 & 3.44 & 3.44 \\
\hline \multirow[t]{3}{*}{ Harina polen } & 6.00 & $34 / 16$ & 20 & & & 6 & 8 & 10 & 20 & 23 & 25 & 3.0 & 3.10 & 3.10 & 3.10 & 3.10 & 3.20 \\
\hline & & & & 25 & & 6 & 8 & 10 & 20 & 23 & 25 & 3.0 & 3.10 & 3.00 & 3.00 & 3.00 & 3.10 \\
\hline & & & & & 30 & 6 & 8 & 10 & 20 & 23 & 25 & 3.0 & 3.20 & 3.20 & 3.20 & 3.20 & 3.20 \\
\hline \multirow[t]{3}{*}{ Harina polen } & 7.00 & $34 / 16$ & 20 & & & 6 & 8 & 10 & 20 & 23 & 25 & 3.6 & 3.40 & 3.50 & 3.50 & 3.45 & 3.50 \\
\hline & & & & 25 & & 6 & 8 & 10 & 20 & 23 & 25 & 3.6 & 3.40 & 3.30 & 3.40 & 3.40 & 3.40 \\
\hline & & & & & 30 & 6 & 8 & 10 & 20 & 23 & 25 & 3.6 & 3.45 & 3.50 & 3.50 & 3.45 & 3.55 \\
\hline
\end{tabular}

Al evaluar los volúmenes obtenidos del pan blanco y determinando que parámetros influyen más sobre la formulación al final se determinaron las siguientes cantidades para su elaboración: Harina de trigo $100 \mathrm{~g}$, Harina de polen 5 $\mathrm{g}$, levadura en polvo $1.2 \mathrm{~g}$, azúcar $2.5 \mathrm{~g}$, sal $1.7 \mathrm{~g}$, margarina $2 \mathrm{~g}$, jarabe agua: miel 50ml (34/16), 1 yema de huevo para barnizar. Se estandarizan parámetros de fermentación a 30 minutos con $40^{\circ} \mathrm{C}$ dentro de una cámara de Fermentación (Brasmig, Brasil) a menor tiempo no aumenta volumen, con una segunda fermentación a la misma temperatura nuevamente en la cámara de fermentación durante 6 minutos a más tiempo no existe más aumento de volumen. El tiempo de horneo se establece a 25 minutos a $190^{\circ} \mathrm{C}$ mayor tiempo influye en una miga dura, textura rígida y sabor detectable de polen.

Una parte de la población es alérgica al polen pero al ser consumido en grano en alimentos o bebidas este efecto desaparece o no se manifiesta en el organismo. Las abejas no consumen polen fresco, esta recolecta los granos de polen transformándolos a granos hidratados e hinchados en comparación con granos de polen frescos debido a la adición de néctar y secreciones glandulares (GHOSH; JUNG, 2015). En pan blanco, pan de dulce o bebidas que contienen polen como agregado para fortificar dichos alimentos por contener un rango de proteína del 16 al $24 \%$ y un balance de aminoácidos que cumple con el patrón (OMS/FAO/UNU 2007), siendo el aminoácido limitante el triptófano para algunos pólenes de flores pero conteniendo una buena cantidad de lisina para ser considerado un nutrimento en la salud humana. Al sustituir harina de polen en un 5 al $10 \%$ para harina de trigo.

En el pan blanco se incrementa el contenido de proteína de este pan en más del $12 \%$ por ciento, por lo que será factible formular un pan con mayor contenido de proteína a través del incremento de harina de polen encontrando como no detectar el sabor amargo del polen en gran cantidad (SAMPATH; CHELEUI, 2017; ZULUAGA et al., 2016; CONTE, 2016; COSTA et al., 2017; MARTINEZ et al., 2017; COSTA et al., 2017).

Formulaciones de pan blanco esférico a base de harina de trigo integral y con harina integral de arroz a diferentes concentraciones establecen tiempos de horneados similares (23-28 minutos), mayor cantidad de agua (60-65 ml), tiempos de fermentación menores (15 minutos) para mayor cantidades de sustitución de harina de trigo por harina integral de arroz, galletas, pastel y bísquet son fortificado con harina seca de plátano (20\%) utilizan tiempos de fermentación de 15 minutos, 10 minutos en la segunda fermentación con 10 minutos a $200^{\circ} \mathrm{C}$ tiempo de horneado. Una mezcla de harinas libre de gluten para panificación y otros ingredientes que incluyen polen con variación de porcentajes de 1 a $5 \%$, fermentan a $30^{\circ} \mathrm{C}-90 \% \mathrm{HR}$, tiempo de fermentación $200^{\circ} \mathrm{C}$ por 70 minutos y enfriado por 2 horas a temperatura ambiente, establece a que a mayor cantidad de polen se incrementa el volumen de la mezcla en 3 y $4 \%$ de adición presentan expansión del área durante el proceso de fermentación con volumen específico más alto después del horneado, a mayor adición ya no influye en el volumen especifico (FIGURA 1). Investigaciones que difieren al trabajo realizado en pan blanco adicionado con harina de polen como mayor tiempo en la fermentación de la masa, no usan miel en el agua para obtener la masa, Temperaturas de horneo más bajas, tiempos sin polen menores y con polen mayores en más del doble de tiempo de horneo (SALA; HAROS, 2016, 2017; CONTE et al., 2018).

Figura 1.Masa obtenida con harina de polen y trigo.

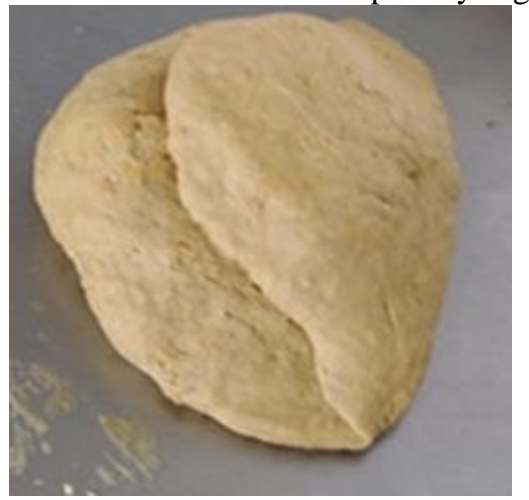

La prueba sensorial de preferencia nos señala que a $80 \%$ le agrado el pan elaborado y el $20 \%$ no lo acepta por poseer un sabor amargo dado por el polen, igualmente por el color (café-verdoso) no característico de la migaja (Figura 2) puede ser atribuible a la harina de polen. Para corregir este nivel de desagrado se corrige con la adición de miel con agua en la preparación, formando un jarabe de 26-28\% Brix en sustitución del agua normal para el pan blanco control, mejorando las propiedades elásticas, textura y miga menos compacta incluso en el pan ya horneado se observa con textura blanda, miga uniforme de aire y mejora del sabor. 
Figura 2. Masa salada cruda y Pan Horneado con harina de polen y trigo.

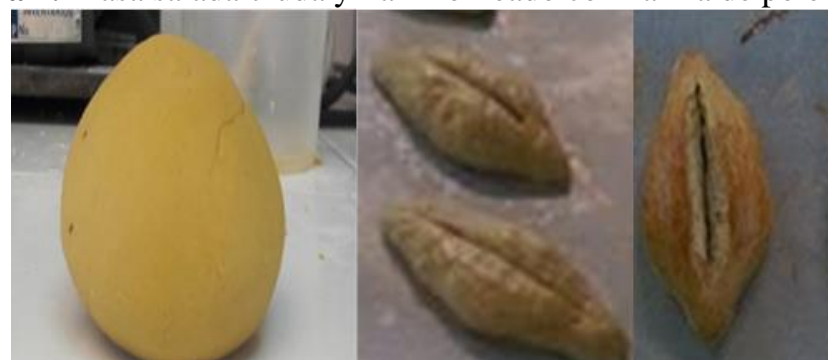

Los bolillos o tortas presentados en la evaluación sensorial de aceptación se les proporciono un pedazo de $3.5 \mathrm{~g}$. en promedio con textura suave, color no común, olor característico, sabor ligero a dulce con una altura en el centro del pan de $3.8 \mathrm{~cm}$ y diámetro en el caso de la torta de $7 \mathrm{~cm}$ en crudo y ya cocido de $8 \mathrm{~cm}$, en el bolillo diámetro en crudo de $5 \mathrm{~cm}$ y ya cocido de $6 \mathrm{~cm}$ en el cual tanto la fermentación segunda como el horneo hacen que se esponje de manera general y una migaja menos apretada, pero influye el color poco agradable del migajón.

Los resultados obtenidos de la evaluación sensorial nos indican que la cantidad de polen adicionada en forma de harina al pan blanco es una cantidad muy pequeña 5 a $7 \%$ que para el consumidor no logra definir sensorialmente que es o que contiene de diferente el alimento presentado (Tabla 2) (Figura 3), el grafico mostrado permite establecer que para la apariencia general, ligera diferencia en olor e inclusive el color en el cual el migajón posee un ligero color oscuroverdoso no les es desagradable, el sabor tampoco difiere por lo que la de mayor cantidad de harina de polen de la de menor no es detectable. En donde sí se establece una ligera diferencia sensorial es en la consistencia general que va de menor a mayor aunque estadísticamente no hay significancia al $5 \%$, por lo que el pan blanco con mayor cantidad de harina de polen es menos agradable que a menor cantidad la cual tiene mejor consistencia en el panelista y estadísticamente el mejor es del 5\% de harina de polen para todas las características. Todas las formulaciones son aceptadas como gusta ni disgusta (3.0) por lo que en general es aceptado sensorialmente el pan elaborado con harina de polen. $\mathrm{Al}$ sustituir harina de trigo por otras harinas ya sea para fortificar o bien solo modificar consistencia y sabor en un pan blanco las características sensoriales son afectadas.

Tabla 2. Evaluación sensorial de aceptación de pan blanco con harina de trigo-polen.

\begin{tabular}{lccccc}
\hline $\begin{array}{c}\text { H. de } \\
\text { Polen \% }\end{array}$ & Color & Olor & Consistencia & textura & Sabor \\
\hline Muestra 7 & $3.1 \mathrm{a}$ & $3.58 \mathrm{a}$ & $2.86 \mathrm{a}$ & $3.18 \mathrm{a}$ & $3.34 \mathrm{a}$ \\
Muestra 6 & $3.2 \mathrm{a}$ & $3.62 \mathrm{a}$ & $3.28 \mathrm{a}$ & $3.18 \mathrm{a}$ & $3.48 \mathrm{a}$ \\
Muestra 5 & $3.14 \mathrm{a}$ & $3.72 \mathrm{a}$ & $3.24 \mathrm{a}$ & $3.12 \mathrm{a}$ & $3.58 \mathrm{a}$ \\
\hline $\begin{array}{l}\mathrm{a}=\text { no existe diferencia al (alfa }=0.5) \\
\text { (alfa=0.5). }\end{array}$ & & & & &
\end{tabular}

Tradicional cadena alimenticia en tanto los fabricantes emprendedores se propongan reemplazar las granjas y las fábricas por los laboratorios. Un importante aspecto para captar a los consumidores será que los productos aportan aceptables sustituciones de sus contrapartes cosechadas. En este sentido, una cuarta parte de los consumidores en el Reino Unido coinciden en que les resultan atractivos los sustitutos de alimentos que resultan similares a lo real en términos de sabor, textura y/o apariencia. Por último, la tecnología también podría utilizarse para diseñar alimentos y bebidas

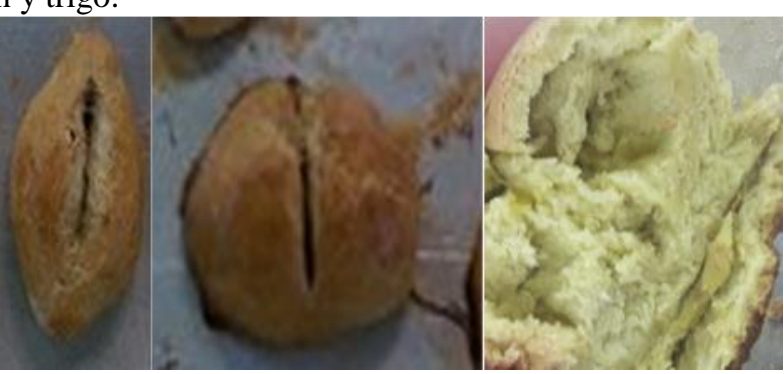

intrínsecamente más nutritivos, lo que ampliaría el público que acepta los productos elaborados científicamente y sumarse a consumidores preocupados por su nutrición (CONTE, 2016; BALCH, 1997; GHOSH et al., 2015; INDUSTRIA ALIMENTARIA, 2015; MURCIA, 2017; MINTEL, 2018).

Figura 3. Análisis sensorial de aceptación con harina de polen al 5, 6 y $7 \%$.

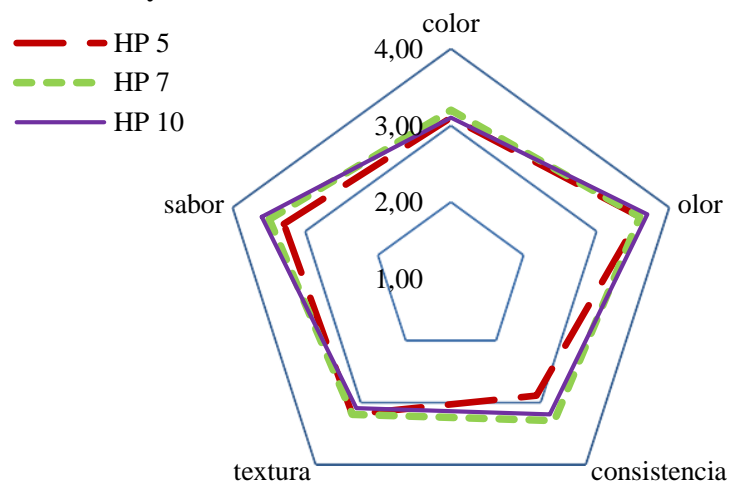

\section{CONCLUSIONES}

Se establece una formulación adecuada del Pan Blanco con harina de trigo-polen, estandarizando ingredientes y sustituyendo el agua por un jarabe de miel-agua (26-28 ${ }^{\circ}$ Brix). Por medio de evaluación sensorial se establece un rango de adición de harina de polen de 1 a $10 \%$ cantidades mayores son detectados sensorialmente por olores, colores y sabores no propios del alimento. La metodología de panificación del producto es de 30 minutos tiempo de fermentación a $40^{\circ} \mathrm{C}$, con 6 minutos de reposo ambiente y temperatura de horneo a $190^{\circ} \mathrm{C}$ por 25 minutos para $100 \mathrm{~g}$. de Masa.

El pan blanco cuando se incorpora en diferentes concentraciones con harina de polen es sensorialmente aceptado en todos los atributos sin haber diferencia significativa, excepto con mejor sabor al $10 \%$ y consistencia al $7 \%$ por lo que el pan obtenido se puede incorporar de 5 a $10 \%$ sustituyendo la harina de trigo en dichas cantidades cuando es elaborado. El Pan Blanco como producto puede tener viabilidad comercial así como también al apicultor un subproducto que pudiera tener alternativa de uso propio o bien de obtener ganancias extras al comercializarlo.

\section{REFERENCIAS}

ACERCA, Organización civil de agricultura y ganadería. Situación actual y perspectivas de la apicultura en México. Claridades Agropecuarias. Agencia de Servicios a la Comercialización y Desarrollo de Mercados Agropecuarios. No. 199 -Marzo, 2010. 
ANPROPAN, Asociación Nacional de proveedores profesionales de la industria del pan, repostería y similares S.A. de C.V-. Industria de la panificación, datos estadísticos. Feria Internacional de la industria del pan, MEXIPAN. 14a . Edición. 2016. Feria internacional de la industria del pan, MEXIPAN. 14 ${ }^{\text {a }}$. Edición. 2016.

AVILÉS-SACOTO, S.; COOK, W. D.; IMANIRAD, R.; ZHU, J. Two-stage network DEA: When intermediate measures can be treated as outputs from de second state. J. of the Operational Research Society. Vol. 6, Issue11; 18681877, 106. 2015.

BALCH, P. A. Recetas nutritivas que curan. New York, Ed.Avery.1997.

CAMACHO-GONZÁLEZ G. Y.; SALGADO-CRUZ, M. LA P; CALDERÓN D. G.; DÍAZ-RAMÍREZ M.; GONZÁLEZ L. L. B. Optimización de un pan dulce tipo "concha" adicionado de hojas de stevia y neem. Investigación y Desarrollo en Ciencia y Tecnología de Alimentos. Vol. 2. 334-339. 2017.

CANAINPA, Cámara Nacional de la Industria Panificadora y Similares de México-. Situación del sector de panificados en México. 2017.

CANAINPA, Cámara Nacional de la Industria Panificadora y Similares de México-. Situación del sector de panificados en México.2016.

CANAINPA, Cámara Nacional de la Industria Panificadora y Similares de México-. Situación del sector de panificados en México.. El mundo del pan. 2016

CANIMOLT, Cámara nacional de molineros y productores de trigo-. Reporte estadístico 2015 con información al 2016. Cámara Nacional de la industria molinera del trigo. Trámite reserva SEP-INDAUTOR. No. 04-2008-060313225100-20. Certificado de solicitud de contenido en trámite. 2016.

CONTE, P.; CARO, A.; BALESTRA, F.; PIGA, A.; FADDA, C. Bee pollen as a functional ingredient in glutenfree bread: A physical chemical, technological and sensory approach. LWT -Food Science and Technology 90.pp. 1-7. 2018.

COSTA, M. C.; MORGANO, M. A.; FERREIRA, M. M.; MILANI, R. F. Analysis of bee pollen constituents from different Brazilian regions: Quantification by NIR spectroscopy and PLS regression. LWT - Food Science and Technology 80.pp.76-83. 2017.

ESPIGUERO, M. Situación actual de la panificación en México en feria alimentaria. FESTIPAN Organizado por Mexipan.

2016

<http://www.festipan.com/singlepost/2016/08/15/Situaci\%C3

$\%$ B3n-actual-de-la-industr\%C3\%ADa-de-la-

panificaci\%C3\%B3n-en-M\%C3\%A9xico>.
ESPINOSA, M. J. Evaluación sensorial de los alimentos. Ministerio de educación superior de cuba. Ed. Universitaria. 2007.

FAO/WHO/OUN, Organización de la naciones unidas para la agricultura y la alimentación/Organización mundial de la salud/Organización de naciones unidas-. Protein and amino acid requirements in human nutrition. Report of a Joint WHO/FAO/UNU Expert Consultation. WHO Technical Report Series No.935 WHO. 2007.

FLECHA, M. Procesos y técnicas de panificación. Sin editorial. 2015. <http://www.academia.edu/30170096/PROCESOS_Y_TECN ICAS_DE_PANIFICACION_Manuel_Flecha>

GOUDA, T. Effectiveness of Some Fortified Nutritional Products with Sun Dried Banana Peel Status of Faculty Education Students in Nujran. J Food Process Technol: 8:12. 2017. 10.4172 / 2.157-7.110, 1000705

GHOSH, S.; JUNG, C. Nutritional evaluation of commercially available pollen patties in Korea. J. Apic. 30, 155-160. 2015.

IMANIRAD, R.; AVILÉS-SACOTO, S.; COOK, W.; ZHU, J. Partial input to output impact in DEA: The case de DMUspecific impacts. European Journal of Operational Research, vol. 244, Issue 3, 837-844. 2013.

INDUSTRIA ALIMENTARIA. Tendencias en panificación 2015. Al día-Boletín electrónico-Industria alimenticia. Febrero. 2015.

KIELISZEK, M.; PIWOWAREK, K.; KOT, A. M.; BŁAŻEJAK, S.; CHLEBOWSKA-ŚMIGIEL, A.; WOLSKA, I. Pollen and bee bread as new health-oriented products: A review. Trends in Food Science \& Technology 71.pp. 170180. 2018.

KRYSTYJAN, M.; GUMUL, D.; ZIOBRO, R.; KORUS, A. The fortification of biscuits with bee pollen and its effect on physicochemical and antioxidant properties in biscuits. LWT - Food Science and Technology. v. 63, n. 1, p.640-6, 2015.

MAMMA, D.; KOURTOGLU, E; CHRISTAKOPOULUS, P. Fungal multienzyme production on industrial by-products of the citrus-processing industry. Bioresourse Technology. 99; 2373:2383. 2008.

MARTÍNEZ, R. A.; SCHVEZOV, N.; BRUMOVSKY, L. A.; PUCCIARELLI, R. A. B. Influence of temperature and packaging type on quality parameters and antimicrobial properties during Yateí honey storage. Food Science and Technology, Campinas. 2017. 10.1590/1678-457X.17717.

MINTEL, J. Z. 5 Grandes tendencias en el mercado a \& b. Énfasis Alimentaria-Marzo. Tomado del Global Food \& Drink Trends. 2018.

MURCIA, J. L. Tendencias internacionales en panadería y bollería, Pan artesano y masas congeladas confluyen en una 
oferta tan variada como atractiva. Distribución y Consumo 48: Vol. 2. 2017.

NATIONAL HONEY BOARD -NIH- . I am here to tell you the bear facts about honey. A Reference Guide from the Honey Board Research Summary. 2010.

NICOLSON, S.W. Digestibility and nutritional value of fresh and stored pollen for honey bees (Apis mellifera scutellata). Journal of Insect Physiology. 10.1016/j.jinsphys.2017.12.008. 2017.

OLIVEIRA, A. P. Dynamic Phosphoproteomics reveals TORC-dependent regulation of yeast nucleotide and amino acid biosynthesis. Sci Signal 374: rs4. 2015.

OPENSHAW, R. La dieta de batidos verdes. Canada: Ulysses Press. 2015.

POPOV-RALJIĆ， J. V.; LALIČIĆ-PETRONIJE， J. G.; GEORGIJEV, A. S.; POPOV, V. S.; MLADENOVIC, M. A. Sensory Evaluation of Pralines Containing Different Honey Products. Sensors. 10; 7913-7933. 2010. 10.3390/s100907913. 2010.

SAGARPA, Secretaria de ganadería y recursos para la agricultura-. Boletín Notiabeja, estadísticas. 13-1. Pag.6-9. 2013.

SAGARPA, Secretaria de ganadería y recursos para la agricultura-. Mercado apícola y Exportaciones de miel. Documento Oficial. 2016. <http://www.sagarpa.gob.mx/saladeprensa/2012/Paginas/201 5B096.aspx.>

SAGARPA, Secretaria de ganadería y recursos para la agricultura-. Precios de la miel por región. Coordinación general de ganadería. Programa nacional para el control de la abeja africana. Notiabeja-enero-febrero. 2015.
SALAS, M. M.; HAROS, M; Evaluation of the technological, nutritional and sensory qualities of bakery products produced with partial substitution of the wheat flour by whole rice flour. Braz. J. Food Technol., Campinas, v. 19, e2016002. 2016.

GHOSH, A. S.; JUNG, C. H.; Nutritional value of beecollected pollens of hardy kiwi, Actinidia argute (Actinidiaceae) and oak, Quercus sp. (Fagaceae). Journal of Asia-Pacific Entomology 20. pp. 245-251. 2017.

SANTACRUZ, V. C; SANTACRUZ, V. V.; HINOJOSA M. J.; ZAMORA, L. M. Retos y perspectivas de la agricultura y alimentos en México. Fomento Editorial BUAP, México. pp. 83-91. 2016.

SAVAL, S. Aprovechamiento de residuos agroindustriales: pasado, presente y futuro. Revista de la sociedad mexicana de biotecnología y bioingeniería A.C (en línea). 16:2, pp.14-46. 2012.

WRIGLEY, C. W. Wheat: An Overview of the Grain That Provides. Our Daily Bread: 105-110. 2016.

ZAVAlA, O. J. A.; COLOMO, G. I.; HIGUERA P. J. El análisis sensorial de miel y la formación de un panel de cata entre los apicultores. Memorias $23^{\circ}$ Congreso Internacional de Actualización Apícola. XV CONGRESO ANMEA. pp. 5962. 2014.

ZULUAGA, C.; MARTÍNEZ, A.; FERNÁNDEZ, J.; LÓPEZ-BALDÓ, J.; QUILES, A. ; RODRIGO, D. Effect of high pressure processing on carotenoid and phenolic compounds, antioxidant capacity, and microbial counts of bee-pollen paste and bee-pollen-based beverage. Innovative Food Science and Emerging Technologies 37. Pp. 0-17. 2016. 